\title{
Processing Influence on Molecular Assembling and Structural Conformations in Silk Fibroin: Elucidation by Solid-State NMR
}

\author{
E. Callone $e^{\dagger, t}, S$. Dire $^{\dagger, t}, X . H u^{\S}$, and A. Motta ${ }^{+,} \mathcal{\xi}^{\prime \cdots, \#}$
}

AUTHOR ADDRESS.

$\dagger$ Department of Industrial Engineering and \$"Klaus Mueller" Magnetic Resonance Laboratory, Department of Industrial Engineering, University of Trento, Via Sommarive 9, 38123 Trento, Italy

$\perp$ BIOtech Research Center, University of Trento, Via delle Regole 101, 38123 Mattarello (TN), Italy

॥Trento Unit, European Institute of Excellence on Tissue Engineering and Regenerative Medicine, Italy

\#Trento Research Unit, INSTM, Trento, Italy

§Department of Physics \& Astronomy, Biomedical \& Translational Sciences, Rowan University, 201 Mullica Hill Road, Glassboro 08028, New Jersey, United States

KEYWORDS. Solid state NMR, Processing, Silk Fibroin, FTIR, Secondary conformation, 3-fold helix 


\section{Biomacromolecules Supporting Information}

An example of the profile fitting of $\mathrm{CF}_{\mathrm{WV}}$ peaks 1 and $\mathbf{9}$ taking into account an extra component with respect to the other samples is offered in Figure S1 for both resonances.
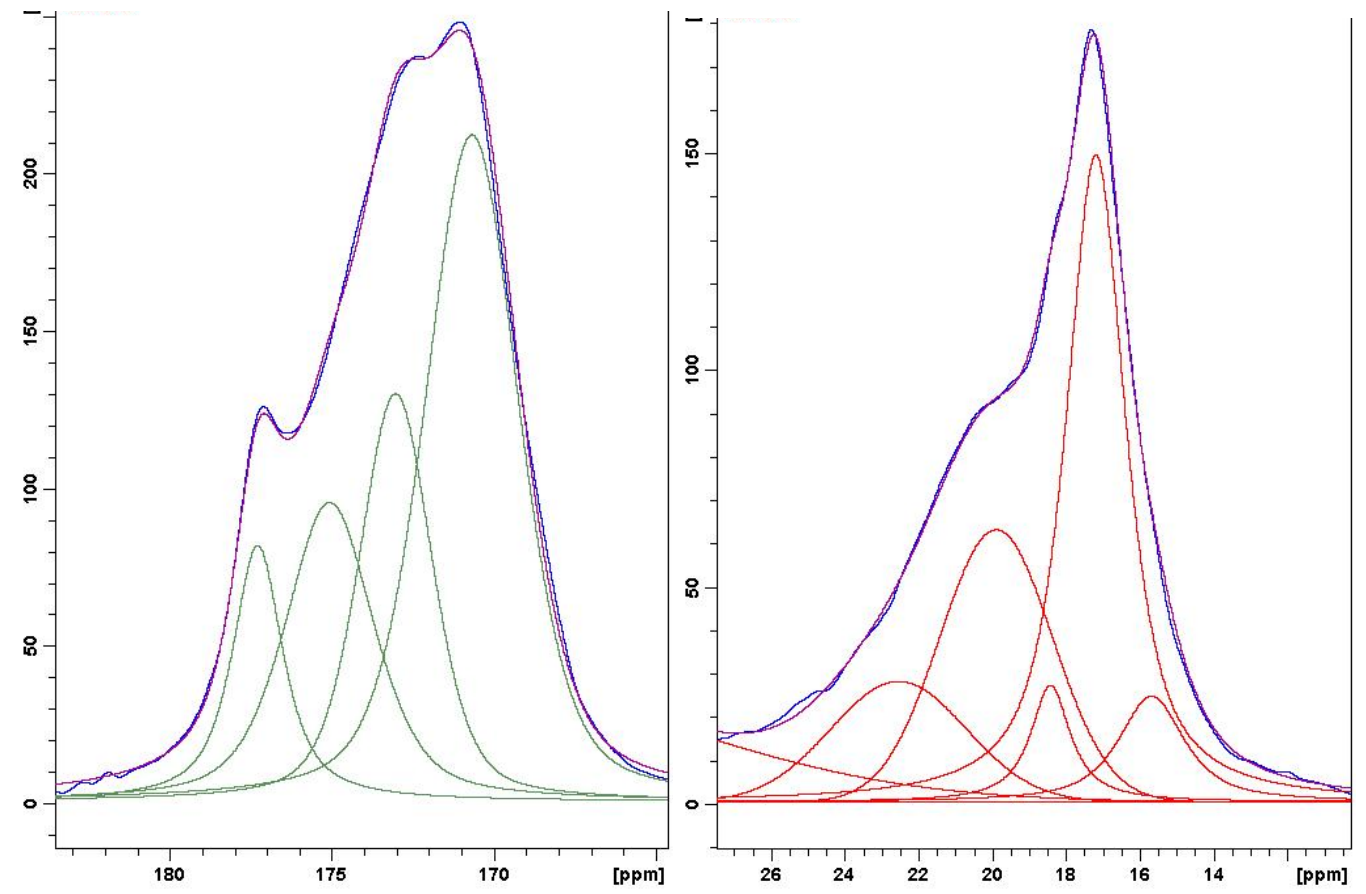

Figure S1. Peak 1 and 9 profile fitting with four and five components, respectively.

The ATR spectra can be treated in a similar way applying a profile fitting on the amide I band, according to Hu et al. ${ }^{12}$ The superimposition of all the spectra (Figure S2) highlights that the CFWV sample gives rise to a band with a quite different shape. As a matter of facts that spectrum is better deconvoluted with an extra component placed at $1646.1 \mathrm{~cm}^{-1}$, as shown in Figure S3.

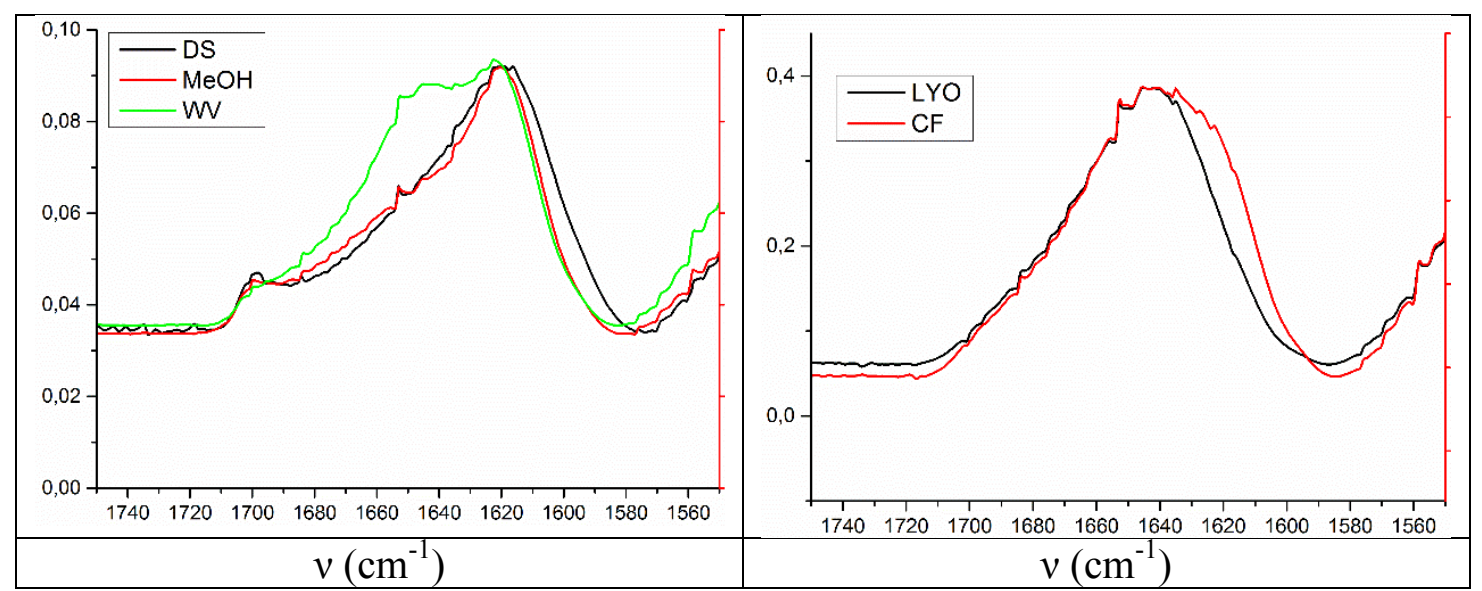

Figure S2. Superimposition of amide I bands of all the samples. 


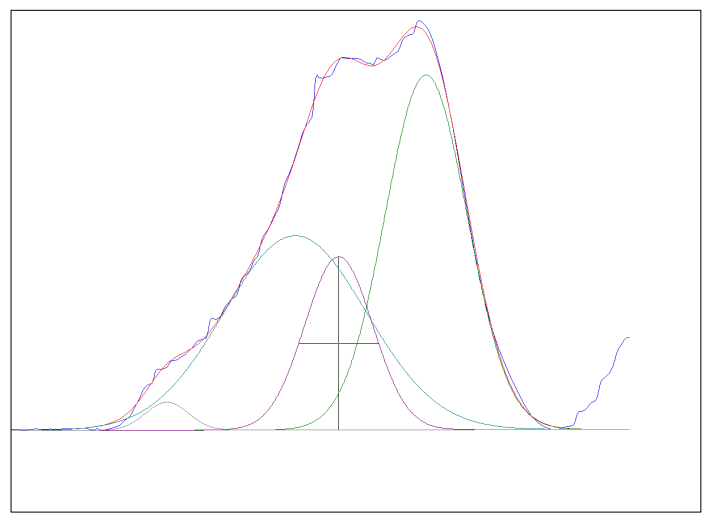

Figure S3. Profile fitting of the amide I band of the $\mathrm{CF}_{\mathrm{WV}}$ sample with four components.

Table S1. Peak assignment and quantitative data according to the profile fitting of the amide I IR band.

\begin{tabular}{|c|c|c|c|c|c|c|c|c|c|c|}
\hline & \multicolumn{2}{|c|}{ DS } & \multicolumn{2}{|c|}{$\mathbf{C F}_{\mathrm{MeOH}}$} & \multicolumn{2}{|c|}{$\mathrm{CF}_{\mathrm{WV}}$} & \multicolumn{2}{|c|}{ CF } & \multicolumn{2}{|c|}{ LYO } \\
\hline & $v\left(\mathrm{~cm}^{-1}\right)$ & $\% \mathbf{A}$ & $v\left(\mathrm{~cm}^{-1}\right)$ & $\% A$ & $v\left(\mathrm{~cm}^{-1}\right)$ & $\% \mathrm{~A}$ & $v\left(\mathrm{~cm}^{-1}\right)$ & $\% A$ & $v\left(\mathrm{~cm}^{-1}\right)$ & $\% A$ \\
\hline Turns & 1690 & 14.5 & 1687.37 & 13.4 & 1696 & 1.7 & 1689.58 & 5.8 & 1687 & 4.4 \\
\hline Random coil & 1653 & 14.1 & 1652 & 34.1 & 1658.9 & 39.7 & 1653.58 & 54. & 1653 & 65.3 \\
\hline & & & & & 1646.1 & 16.7 & & & & \\
\hline$\beta$-sheets/strands & 1621 & 71.4 & 1619.1 & 52.5 & 1620.4 & 41.9 & 1624.94 & 39.8 & 1635.5 & 30.4 \\
\hline
\end{tabular}

